\title{
Celiac Disease-specific Prolamin Peptide Content of Wheat Relatives and Wild Species Determined by ELISA Assays and Bioinformatics Analyses
}

\author{
Gy. GELL ${ }^{1}$, K. KOVÁCS ${ }^{1}$, I. MOLNÁR ${ }^{2}$, Zs. BUGYI ${ }^{3}$ S. TÖMÖSKÖZI ${ }^{3}$ and A. JUHÁSZ ${ }^{1 *}$ \\ ${ }^{1}$ Applied Genomics Department, Agricultural Institute, Centre for Agricultural Research, HAS, \\ Martonvásár, Hungary \\ ${ }^{2}$ Department of Plant Genetic Resources and Organic Breeding, Agricultural Institute, \\ Centre for Agricultural Research, HAS, Martonvásár, Hungary \\ ${ }^{3}$ Department of Applied Biotechnology and Food Science, \\ Budapest University of Technology and Economics, Budapest, Hungary \\ (Received 8 September 2014; Accepted 10 November 2014; \\ Communicated by F. Békés)
}

\begin{abstract}
Enzyme-linked immunosorbent assays (ELISAs) are widely used to determine gluten contamination in gluten-free and low gluten food samples. ELISA assays developed using monoclonal antibodies against known toxic peptides have an advantage in the identification of toxic prolamin content in protein extracts of different food samples, as well as raw materials. R5 and G12 monoclonal antibodies specific for two known toxic peptides used in commercially available gluten ELISA assays were applied to test toxic peptide contents in wheat relatives and wild wheat species with different genome composition and complexity. Although the R5 peptide content showed some correlation with ploidy levels in Triticum species, there was a high variance among Aegilops species. Some of the analysed diploid Aegilops species showed extremely high R5 peptide contents. Based on the bioinformatics analyses, the R5 peptide was present in most of the sulphur rich prolamins in all the analysed species, whereas the G12 epitope was exclusively present in alpha gliadins. High variation was detected in the position and frequency of epitopes in sequences originating from the same species, thus highlighting the importance of genotypic variation within species. Identification of new prolamin alleles of wheat relatives and wild wheat species is of great importance in order to find germplasm for special end-use quality purposes as well as development of food with reduced toxicity.
\end{abstract}

Keywords: ELISA, R5 epitope, G12 epitope, celiac disease, cereal species

\section{Introduction}

The unusual amino acid composition and primary structure of prolamins in cereal grains have a specific role in defining their nutritional and health-related properties (Sampson and Metcalfe 1992). Gluten-related disorders are divided into three main groups, namely autoimmune, allergy, and non-autoimmune allergic disorders (Sapone et al. 2012). Two

* Corresponding author; E-mail: juhasz.angela@agrar.mta.hu; Phone: +36-22-569-531; Fax: +36-22-569-514 
of these disorders are relatively well described; however, further studies are needed to reveal mechanisms related to the diverse symptoms of allergic reactions. The autoimmune disorders include celiac disease (CD) (Anderson and Wieser 2006), gluten ataxia, and dermatitis herpetiformis (Laurièrre et al. 2006). The allergic reactions involve respiratory allergy (Amano et al. 1998), food allergy (Mills et al. 2003), wheat-dependent exercise-induced anaphylaxis (Armentia et al. 1990) and contact urticaria. While characteristic symptoms of celiac disease develop after different receptor proteins (for example HLA DQ) bind to the antigens on the surface of antigen-presenting cells, the development of wheat allergies is mediated more directly by the recognition of allergens by IgE epitopes bound to mast cells (Catassi and Fasano 2008). In celiac disease, the presence of HLA DQ molecules such as DQ2.5, DQ8, DQ2.2 are clear markers for the development of autoimmune symptoms, with modifying effects coming from genetic and environmental factors (Anderson et al. 2000). Patients suffering from celiac disease produce a range of autoimmune responses to several prolamin peptides in wheat, barley and rye products. The nine amino acid-long core regions of these peptides, also called core-epitopes, are processed by the tissue transglutaminase 2 (tTG2) and are presented in their deamidated form by human leucocyte antigen presenting cells (HLA DQ dimers) to the T-cell receptors. There are a number of different conditions that need to occur simultaneously to provoke an autoimmune response (Anderson et al. 2000). Based on the knowledge of genetic, immunologi$\mathrm{cal}$, and biochemical mechanisms of $\mathrm{CD}$, a set of criteria can be defined for the structure of an active celiac disease-specific core-epitope: a) a size of nine amino acids rich in proline and glutamine, $b$ ) the presence of a tissue transglutaminase 2 (tTG) enzyme binding site, and c) presence of amino acids surrounding the core-epitope with defined charge and hydrophobicity (Sollid et al. 2012).

The prolamin superfamily of cereal seeds contains the highest number of harmful proteins of all plant protein superfamilies (Radauer and Breiteneder 2007). Four of its eight member families, namely prolamins, nsLTPs, alpha-amylase/protease inhibitors and $2 \mathrm{~S}$ albumins, contain peptides resulting in different wheat related disorders. Among these, real prolamins, namely HMW- and LMW-glutenins, and alpha-, gamma- and omegagliadins, have significant roles in triggering the mechanisms of celiac disease. This fact is largely due to their high sequence similarity, the presence of shorter or longer repetitive regions in their primary protein structures and the significantly unbalanced amino acid composition, as these proteins are rich in proline and glutamine residues (Breiteneder and Radauer 2004).

Aegilops species are excellent resources for pre-breeding to broaden agronomically important traits of hexaploid bread wheat species. They serve as valuable sources of biotic and abiotic stress resistance (Schneider et al. 2008) and enhanced micronutrient content (Farkas et al. 2014). They may therefore be beneficial in preventing certain forms of chronic diseases (Santra et al. 2013). A number of studies have been published that are focusing on the identification of new storage protein alleles from wild wheat relatives, mainly with the aim of developing wheat sources with targeted end-use quality. Next to the quality-related issues, several studies have focused on the assessment of toxicity of wheat genome donors, such as Triticum monococcum $\left(\mathrm{A}^{\mathrm{m}}\right.$ genome) and Aegilops 
tauschii (D) or Triticum urartu ( $\left.\mathrm{A}^{\mathrm{u}}\right)$. Most of these studies investigated single protein families, such as alpha- or gamma-gliadins (Molberg et al. 2005; van Herpen et al. 2006). However, relatively little is known about the allergenic and toxic nature of wild wheat species, such as different Aegilops species.

Enzyme-linked immunosorbent assay (ELISA) is a relatively simple and straightforward methodology to determine the level of particular prolamin components in protein extracts or food samples. The conversion of gliadin or total prolamin concentration to gluten content, expressed in ppm $(\mathrm{mg} / \mathrm{kg})$ is a generally used value to decide whether a product can be labeled gluten-free (Codex standard 118-1979). The reliability of the obtained gluten content highly depends on the types and expressed amounts of specific peptide sequences or proteins the antibodies are raised against. Some of the gluten-specific ELISA kits, such as the Morinaga Wheat Gliadin kit (Morinaga Institute of Biological Science, Japan), use polyclonal antibodies ( $\mathrm{pAb}$ ) while other tests were developed using monoclonal antibodies $(\mathrm{mAb})$ e.g. Skerritt antibody, specific for omega gliadins and HMW glutenins (Skerritt et al. 1988) coupled with different extraction methods and calibration materials. Enhanced detection of toxic peptides can be carried out by the use of mAbs raised against known immunoreactive epitopes. Antibodies such as the R5 antibody developed by Valdés et al. (2003) or the G12 mAb (Moron et al. 2008) were specifically developed to detect peptides present in some of the most frequent epitopes with known immune reactivity/immunoreactivity. The $\mathrm{R} 5 \mathrm{mAb}$ was developed against the peptide QQPFP, present in rye secalins, barley hordeins and wheat gliadins. The R5 mAb is also able to recognize homologous epitopes such as LQPFP, QLPYP, QLPTF, QQSFP, QQTFP, PQPFP and QQPYP, although with weaker reactivity (Osman et al. 2001). The monoclonal antibody G12 was developed against the QPQLPY peptide, present in the toxic 33mer of alpha gliadin (LQLQPFPQPQLPYPQPQLPYPQPQLPYPQPQPF), which contains three different core-epitopes, namely PFPQPQLPY, PQPQLPYPQ and PYPQPQLPY, of which two are present in duplicate. Although the presence of the $33 \mathrm{mer}$ is not common in the alpha gliadins, partial sequences are present in many alpha gliadin sequences (van Herpen et al. 2006). Tests based on these epitopes can provide information on the toxic epitope content of the different protein fractions present in the various genotypes. The aim of our study was to use bioinformatics tools and two commercially available ELISA kits developed against known toxic peptides in order to determine toxic protein contents of certain wheat species and wild wheat relatives.

\section{Materials and Methods}

\section{Plant materials}

Seeds of different diploid and tetraploid Triticum and Aegilops species were used in the study to determine celiac disease-related protein content of diploid and tetraploid genomes compared to bread wheat (Table 1). In addition to $\mathrm{A}^{\mathrm{u}}, \mathrm{A}^{\mathrm{m}}, \mathrm{B}$ and $\mathrm{D}$ genome species, some Aegilops species that are often used for pre-breeding purposes, possessing $\mathrm{M}$ and $U$ genomes, were also included in the study. Seeds obtained from the Department of 
Plant Genetic Resources and Organic Breeding (MTA Centre for Agricultural Research, Martonvásár, Hungary), were milled into flour using a Cyclotec Laboratory Mill (FOSS Tecator, Sweden).

Table 1. Species used in the analysis. Genome compositions and protein contents were measured using the Dumas method

\begin{tabular}{lcc}
\hline Species (genotype) & Genome & Protein content (\%) \\
\hline Aegilops comosa (MvGB1039) & $\mathrm{M}$ & 27.66 \\
Aegilops umbellulata (Mv201001) & $\mathrm{U}$ & 26.49 \\
Aegilops tauschii (MvGB589) & $\mathrm{D}$ & 14.93 \\
Aegilops speltoides (MvGB1147) & $\mathrm{S}$ & 23.56 \\
Triticum monococcum (Mv Alkor) & $\mathrm{Am}$ & 19.18 \\
Triticum urartu (MvGB115) & $\mathrm{Au}$ & 23.78 \\
Aegilops biuncialis (MvGB642) & $\mathrm{UM}$ & 23.06 \\
Aegilops geniculata (Ae660/83) & $\mathrm{UM}$ & 24.44 \\
Triticum turgidum ssp. dicoccum (Mv Hegyes) & $\mathrm{AuB}$ & 15.47 \\
Triticum aestivum (Bánkúti 1201) & $\mathrm{AuBD}$ & 13.82 \\
Triticum aestivum (Chinese Spring) & $\mathrm{AuBD}$ & 12.99 \\
\hline
\end{tabular}

\section{Protein content}

Crude protein content of the samples was determined using the Dumas method in triplicate. Protein content was calculated from the nitrogen content using a nitrogen conversion factor of 5.8 .

\section{ELISA assays}

The R5 Ridascreen Gliadin (R-Biopharm, Germany) sandwich enzyme immunoassay and the AgraQuant Gluten G12 (Romer Labs, Austria) sandwich enzyme assay were used to determine the toxic epitope content in the prolamin extracts of different cereal species. Prolamin extracts were prepared in four replicates using the methodology provided by the kit suppliers (Table S1*). To measure gliadin content of wheat flour samples in the range of quantitation, extracts were diluted by 1:5000. ELISAs were performed as outlined in the manuals of the assays provided by the manufacturers.

Results were interpreted by interpolating OD values from the standard values, corrected by the dilution factor used for the flour samples. Calculated gliadin contents determined by the ELISA assays were normalized by the protein content of each genotype. Values obtained for the hexaploid cultivar Chinese Spring were used as an arbitrary benchmark of toxic R5 and G12 peptide content and the obtained gliadin contents were normalized against these values. These relative values were used to compare toxic protein contents of the analyzed genotypes.

* Further details about the Electronic Supplementary Material (ESM) can be found at the end of the article. 


\section{Bioinformatics analyses}

Characteristic prolamin protein sequences, representing different prolamin families for each species, were retrieved from the UniProt database. ProPepper, a database for identification of toxic epitopes and peptides in prolamins (propepper.net) was used to identify storage protein sequences with the R5 and G12 epitopes. Chinese Spring was used as reference hexaploid genotype possessing both G12 and R5 peptides.

\section{Results}

Protein contents of the analyzed species showed high variability, with significantly higher protein contents in Aegilops species (Table 1). When species with different ploidy level are compared, diploid species generally possess higher protein content (above $20 \%$ ), except for Ae. tauschii, which shows similar protein content to Triticum species (13-15\%).

The ELISA results normalized against the protein content showed significantly different levels of mAb specific responses. Using the R5 mAb, the results of the analyzed species varied between 1,007 and 53,101 ppm gliadin (Fig. 1). The T. monococcum and the Ae. comosa genotype contained the least R5 epitope (QQPFP)- containing protein, while the analyzed Ae. speltoides and Ae. umbellulata genotypes resulted in the highest level of measured gliadin. Significantly lower amounts of R5 mAb specific proteins were detected in the tetraploid species. The hexaploid wheat genotypes resulted 30,993 to 40,570 ppm gliadin. In case of the G12 epitope (QPQLPY) no response was detected for the T. mono-

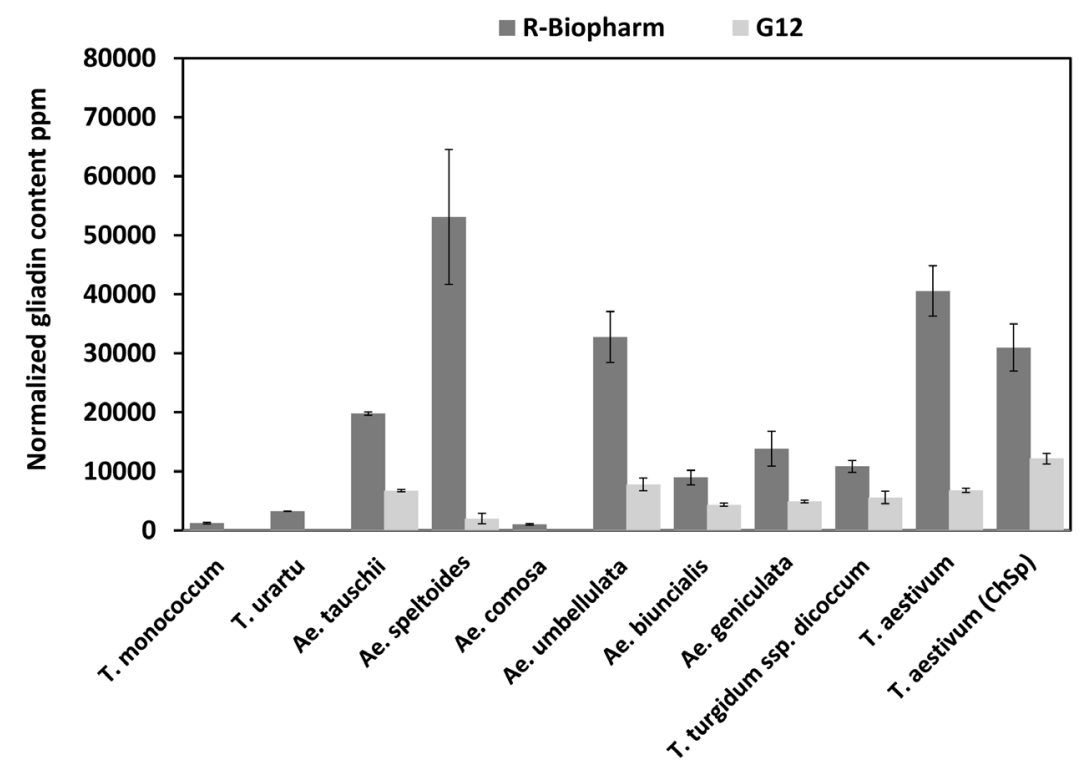

Figure 1. Measured R5 and G12 gliadin contents in diploid, tetraploid and hexaploid cereal species. Dark grey columns represent the values obtained using the R5 mAb assay, light grey columns show results for G12 mAb. Error bars represent standard deviations calculated based on the results of replicates 
coccum and T. urartu genotypes, and the response was below $66 \mathrm{ppm}$ gliadin for the $A e$. comosa. The highest value was seen in T. aestivum cv. Chinese Spring (12,160 ppm gliadin) followed by the Ae. umbellulata genotype (7,786 ppm gliadin).

When the obtained monoclonal epitope-specific protein contents were compared to that of cv. Chinese Spring, R5 mAb-specific gliadin contents were lower than that of cv. Chinese Spring, except for three accessions (Fig. S1). The analyzed Ae. speltoides accession resulted in a 70\% higher R5 mAb-specific protein content. Significantly lower R5 peptide-containing protein contents were measured in the T. monococcum, T. urartu and the Ae. comosa accessions. As for the G12-specific mAb responses, all accessions possessed lower G12 peptide-containing protein levels relative to Chinese Spring.

Bioinformatics analyses were carried out to analyze the occurrence of the analyzed epitopes in the different storage protein families. Altogether 613 storage protein sequences were analyzed, representing all studied species except for Ae. biuncialis, where no prolamin sequence data were found in the public databases. The majority of sequences originated from the Triticum species and Ae.tauschii, and only a few sequences were available for the Aegilops species. Therefore the occurrence of epitope-positive sequences in individual storage protein families was calculated for each species separately (Table S2). The R5 epitope was characteristic in LMW glutenins, and alpha-, gamma- and omega gliadins. Generally, about $25 \%$ of the known LMW glutenin subunits contained the epitope, and it was a sole characteristic of m-type LMW glutenins. The occurrence of the R5 peptide in the alpha gliadin sequences varied between $41.7 \%$ and $100 \%$, with an overall value of $85 \%$. Interestingly, nearly $100 \%$ of the analyzed gamma and omega gliadins contained the epitope. All the epitope-positive omega gliadins belong to the cysteine mutant omega gliadins or LMW-D glutenins. The G12 epitope was exclusively identified in alpha gliadins with a prevalence of 61.1 to $100 \%$.

When the epitopes were mapped to the sequences, the number and position of the epitopes showed high variability. The pattern variations and epitope counts are presented in Table S3. Gamma gliadins showed the highest pattern diversity, with eleven different epitope patterns in T. aestivum, 8 in Ae. tauschii and 7 in T. monococcum. The number of epitopes per protein sequence was 1 or 2 in alpha-gliadins and LMW glutenins, while gamma-gliadins and especially the epitope-positive omega gliadins were rich in this peptide with a maximum of 20 epitopes in one of the omega-gliadin sequences. The G12 epitope was less abundant and the number of pattern variations was relatively low. The position of epitopes was highly conserved, regardless of species. The highest number of pattern variations was present in the hexaploid wheat sequences with a maximum number of 3 epitopes in a single sequence.

\section{Discussion}

ELISA-based gluten tests are widely accepted for the determination of gluten contamination in gluten-free and low-gluten food samples. Depending on the antibodies used, the assays are measuring a specific protein type, a single peptide or a peptide set specific for a group of gluten proteins. Recent in-silico studies proved that all the Osborne fractions may 
contain proteins with toxic epitopes (Juhász et al. 2012a, b), however, their number and expressed amount varies. The number of known toxic celiac disease-related epitopes is continuously growing. Next to the published 9mer core epitopes (Sollid et al. 2012), there are longer peptides with known immunoreactivity, such as the alpha-gliadin 33mer or the 17mer identified in omega-gliadins (Camarca et al. 2009). Many of these epitopes are present in multiple storage protein groups. Therefore, extraction conditions specific for one of the Osborne fractions, such as gliadins or reduced glutenins, will result in partial information about the complete toxic protein content. The use of epitope-specific monoclonal antibodies can enhance our understanding of the toxic epitope content of gluten fractions at a genotypic or species level. The G12 mAb was developed based on the 33mer toxic peptide. The $33 \mathrm{mer}$ is characteristic of alpha-gliadins and contains 5 overlapping epitopes, of which a 6mer peptide, QPQLPY, is used in the AgraQuant Gluten G12 assay. Based on our analyses, the $33 \mathrm{mer}$ was present in only 12 of the 582 alpha-gliadin sequences, all of Chinese origin. However, partial sequences of the $33 \mathrm{mer}$ are characteristic of a significant number of alpha-gliadins. The G12 peptide, which is present in two of the core-epitope sequences of the 33mer, is exclusively characteristic of alpha-gliadins. The absence of this peptide in the other protein families is in concordance with the significantly lower ELISA values compared to that of the R5 mAb. Additionally, the alpha gliadins recognized by the G12 mAb did not represent the entire alpha gliadin fraction. There are alpha gliadin types, especially in the T. urartu and T. aestivum species that do not contain this peptide and none of the epitopes present in the 33mer. Similarly, about $20 \%$ of the known alpha-gliadin sequences in Ae. tauschii and Ae. umbellulata do not contain this peptide.

In contrast, the R5-specific $\mathrm{mAb}$ can bind to proteins from most of the storage protein groups of most of the analyzed species, except for HMW glutenins. No sequence information was available for the Ae. biuncialis species and only one complete LMW glutenin sequence was found for Ae. geniculata. Although the R5 peptide QQPFP was originally identified in a rye omega secalin, it is generally present in gamma gliadins and in about $80 \%$ of alpha-gliadins. It was also mapped to m-type LMW glutenins in most of the species. M-type LMW glutenin subunits are the most variable group of LMW GSs, containing several different subtypes and are encoded on all three genomes of hexaploid wheat. Next to the A, B and D genome species they are also characteristic of the $\mathrm{U}$ and $\mathrm{M}$ genomes. Generally, these types have shorter protein sequences, with shorter repetitive sections, which also means less toxic epitope content, compared to s- and i-type sequences. When epitope counts are considered, the R5 epitope shows much higher variability than the R12 in the different storage protein families. Presence of this conserved peptide is characteristic in most of the prolamins of cereal species, except for HMW glutenin sequences. This finding can be related to the evolutionary development of sulphur-rich prolamin sequences. A possible development of sulphur-rich prolamin proteins, such as the alpha- and gamma-gliadins, LMW glutenins, and cysteine-mutant omega-gliadins, was a result of expansion in the numbers of short repetitive insertions rich in proline and glutamine relative to several ancient prolamin genes (Clarke and Appels 1999). The presence of highly conserved sections in the $\mathrm{N}$ terminal, repetitive and $\mathrm{C}$ terminal domains 
combined with variable sections of the repetitive region led to the development of high prolamin sequence abundance and variance uniquely characteristic of cereal grains. Similarly, high variance in epitope frequency was also seen when sequences from the same protein family were compared. This high QQPFP peptide abundance can be the explanation of the significantly higher measured values in the Ridascreen R5 Gliadin assay. Although neither the R5 nor the G12 antibody is suitable to measure all toxic proteins, they do provide reliable results for the presence of toxic proteins in the different species. However, based on our epitope mapping analyses there is a significant number of sequences that do not contain these peptides (Table S2). Additionally, the R5 peptide was also present in the m-type LMW glutenins (Table S3) that were not represented in the gliadin standard used for calibration of the R5 mAb-specific ELISA assay.

Thus, the tests using R5 and G12 mAbs are underestimating the gluten content of the analyzed samples. The level of this underestimation strongly depends on the expressed amounts of these peptides, which is a factor influenced by the types and number of unique proteins and their expression level. Both the epitope mapping analyses and the ELISA assay results confirm that epitope content of species from the same ploidy level show high variability. While species related to the $\mathrm{A}$ and $\mathrm{M}$ genomes (T. urartu, T. monococcum, Ae. comosa) have the lowest R5 and G12 epitope contents, the U and B genomes in diploid species show higher R5 epitope contents. Even though the highest number of epitopes was found in the cysteine mutant omega gliadins and some gamma gliadins of T. aestivum, these sequence variations were less frequent.

The intended use of ELISA based gluten assays in the food industry is to determine low amounts of gluten in food samples. However, the use of toxic-epitope specific mAbs can be beneficial when toxic protein contents of different cereal species should be determined. Identification of cereal species or protein fractions suitable for special diets is of great importance in the development of food sources for patients. Development of novel celiac disease epitope-specific ELISA tests can result in a more precise determination of the toxic protein content of gluten samples. The ELISA assays performed in this study were used to characterize selected species with different genomic backgrounds. However, the high variation in epitope frequencies of sequences within the same species obtained by bioinformatics analyses highlight the importance of analysis at the genotypic or accession level. Identification of new prolamin alleles of various wheat species and wild relatives is of great importance in order to find germplasm suitable to grow in different environmental conditions and also to find breeding sources suitable for special end-use requirements of consumers with gluten sensitivity.

The joint sequential and immunoanalytical study of cereals and wild wheat relatives has both analytical and clinical implications. First of all, our results provide further confirmation of the considerable variability in expression of celiac-specific peptides of cereals with different genetic backgrounds. Besides, the results show further variability depending on the antibody used. These results highlight the fact that the reliability of immunoanalytical results may be dependent on the variety of the sample and the method of choice. Today, the ELISA methodology is used to determine whether food products intended for celiac patients contain gluten below the regulated threshold levels of 20 and 
$100 \mathrm{ppm}$ gluten for gluten-free and low gluten foods, respectively. Thus, based on our results, it would be important to reveal how this variability affects the analytical results in this low ppm range. It is even more important for the species that are showing lower ELISA gliadin values, or none at all, as in the case of e.g. T. monococcum.

T. monococcum, T. urartu, Ae. comosa were identified to have significantly lower gliadin content as per ELISA methods using antibodies against confirmed toxic epitopes. Although our results can only serve as a pilot study to study the epitope content of wheat relative species some of the analyzed accessions seem to contain remarkably lower levels of gliadin compared to bread wheat. However, these levels are still higher than the $20 \mathrm{ppm}$ gluten level which is required for the gluten-free declaration of a product. Still, these species can be ideal candidates for breeding new varieties with lower toxic-epitope content, this way providing new dietary supplements for celiac patients. However, before that point could be reached, their potentially lower toxicity must be confirmed by in vitro or in vivo studies. Additionally, variability in allergen content and effect of environmental changes on allergen protein content of the different species should be determined. Additionally, effects of food processing and subsequent gastrointestinal digestion on the toxic epitope content, on their biological reactivity and on detectability should be determined.

In spite of raising many new questions, these results are revealing important analytical, clinical and potential product development issues to be addressed in order to improve the quality of life of celiac patients.

\section{Acknowledgements}

This project was supported by the European Union and co-financed by the European Social Fund (grant agreement no. TÁMOP-4.2.2.A-11/1/KONV-2012-0008). Gyöngyvér Gell wishes to thank the support of the MTA Post Doctoral Fellowship. Angéla Juhász was supported by the TÁMOP-4.2.4.A/2-11/1-2012-0001 grant.

\section{References}

Amano, M., Ogawa, H., Kojima, K., Kamidaira, T., Suetsugu, S., Yoshihama, M., Satoh, T., Samejima, T., Matsumoto, I. 1998. Identification of the major allergens in wheat flour responsible for baker's asthma. Biochem. J. 330:1229-1234.

Anderson, R.P., Degano, P., Godkin, A.J., Jewell, D.P., Hill, A.V.S. 2000. In vivo antigen challenge in celiac disease identifies a single transglutaminase-modified peptide as the dominant A gliadin T-cell epitope. Nature (Med) 6:337-342.

Anderson, R.P., Wieser, H. 2006. Medical applications of gluten-composition knowledge. In: Wrigley, C.W., Békés, F., Bushuk, W. (eds), Gliadin and Glutenin. In: The Unique Balance of Wheat Quality. AACCI Press. St. Paul, MN, USA, pp. 387-409.

Armentia, A., Martin-Santos, J.M., Blanco, M. 1990. Exercise induced anaphylaxis reaction to grain flows. Ann. Allergy 65:149-151.

Breiteneder, H., Radauer, C. 2004. A classification of plant food allergens. J. Allergy Clin. Immunol. 113:821-830.

Camarca, A., Anderson, R.P., Mamone, G., Fierro, O., Facchiano, A., Costantini, S., Zanzi, D., Sidney, J., Auricchio, S., Sette, A., Troncone, R., Gianfrani, C. 2009. Intestinal T cell responses to gluten peptides are 
largely heterogeneous: Implications for a peptide-based therapy in celiac disease. J. Immunol. 182:4158-4166.

Catassi, C., Fasano, A. 2008. Celiac disease. In: Arendt, E.K., Dal Bello, F. (eds), Gluten-Free Cereal Products and Beverages. Academic Press, San Diego, CA, USA, pp. 1-28.

Clarke, B.C., Appels, R. 1999. Sequence variation at the Sec-1 locus of rye, Secale cereale (Poaceae). Plant Syst. Evol. 214:1-14.

Codex Standard for Foods for Special Dietary Use for Persons Intolerant to Gluten - CODEX STAN 118 - 1979, adopted in 1979; amended in 1983; revised in 2008, Codex Alimentarius, International Food Standards. Rome, Italy.

Farkas, A., Molnár, I., Dulai, S., Rapi, S., Oldal, V., Cseh, A., Kruppa, K., Molnár-Láng, M. 2014. Increased micronutrient content $(\mathrm{Zn}, \mathrm{Mn})$ in the $3 \mathrm{Mb}(4 \mathrm{~B})$ wheat - Aegilops biuncialis substitution and $3 \mathrm{Mb} .4 \mathrm{BS}$ translocation identified by GISH and FISH. Genome 57:61-67.

Juhász, A., Gell, Gy., Sebestyén, E., Haraszi, R., Tamás, L., Balázs, E. 2012a. Brachypodium distachyon as a model for defining the allergen potential of non-prolamin proteins. Funct. Integr. Genomics 12:439-446.

Juhász, A., Gell, Gy., Békés, F., Balázs, E. 2012b. The epitopes in wheat proteins for defining toxic units relevant to human health. Funct. Integr. Genomics 12:585-598.

Laurièrre, M., Pecquet, C., Bouchez-Mahiout, I., Snegaroff, J., Bayrou, O., Raison-Peyron, N., Vigan, M. 2006. Hydrolyzed wheat proteins present in cosmetics can induce immediate hypersensitivities. Contact Dermatitis 54:283-289.

Mills, E.N.C., Madsen, C., Shewry, P.R., Wichers, H.J. 2003. Food allergens of plant origin - their molecular and evolutionary relationships. Trends in Food Sci. Technol. 14:145-156.

Molberg, O., Uhlen, A.K., Jensen, T., Flaete, N.S., Fleckenstein, B., Arentz-Hansen, H., Raki, M., Lundin, K.E., Sollid, L.M. 2005. Mapping of gluten T-cell epitopes in the bread wheat ancestors: implications for celiac disease. Gastroenterology 128:393-401.

Morón, B., Cebolla, Á., Manyani, H., Álvarez-Maqueda, M., Megías, M., Thomas, M. del C., López, M.C., Sousa, C. 2008. Sensitive detection of cereal fractions that are toxic to celiac disease patients by using monoclonal antibodies to a main immunogenic wheat peptide. Am. J. Clin. Nutr. 87:405-414.

Osman, A.A., Uhlig, H.H., Valdes, I., Amin, M., Mendez, E., Mothes T. 2001. A monoclonal antibody that recognizes a potential coeliac-toxic repetitive pentapeptide epitope in gliadins. Eur. J. Gastroent. Hepatol. 13:1189-1193.

Radauer, C., Breiteneder, H. 2007. Evolutionary biology of plant food allergens. J. Allergy Clin. Immun. 120:518-525.

Sampson, H.A., Metcalfe, D.D. 1992. Food allergies. J. Amer. Med. Assoc. 268:2840-2844.

Santra, M., Matthews, S.B., Thompson, H.J. 2013. Development of a core collection of Triticum and Aegilops species for improvement of wheat for activity against chronic diseases. Agriculture and Food Security 2:4.

Sapone, A., Bai, J.C., Ciacci, C., Dolinsek, J., Green, P.H.R., Hadjivassiliou, M., Kaukinen, K., Rostami, K., Sanders, D.S., Schumann, M., Ullrich, R., Villalta, D., Volta, U., Catassi, C., Fasano, A. 2012. Spectrum of gluten-related disorders: Consensus on new nomenclature and classification. BMC Medicine 10:13.

Schneider, A., Molnár, I., Molnár-Láng, M. 2008. Utilisation of Aegilops (goatgrass) species to widen the genetic diversity of cultivated wheat. Euphytica 163:1-19.

Skerritt, J.H., Lew, P.H., Castle, L.S. 1988. Accumulation of gliadin and glutenin polypeptides during development of normal and sulphur-deficient wheat seed: Analysis using specific monoclonal antibodies. J. Exp. Bot. 39:723-737.

Sollid, L.M., Qiao, S.W., Anderson, R.P., Gianfrani, C., Koning, F. 2012. Nomenclature and listing of celiac disease relevant gluten T-cell epitopes restricted by HLA-DQ molecules. Immunogenetics 64:455-460.

Valdés, I., García, E., Llorente, M., Méndez, E. 2003. Innovative approach to low-level gluten determination in foods using a novel sandwich enzyme-linked immunosorbent assay protocol. Eur. J. Gastroenterol. 15:465-474.

van Herpen, T.W.J.M., Goryunova, S.V., van der Schoot, J., Mitreva, M., Salentijn, E.M.J., Vorst, O., Schenk, M.F., van Veelen, P.A., Koning, F., van Soest, L.J.M., Vosman, B., Bosch, D., Hamer, R.J., Gilissen, L.J.W.J., Smulders, M.J.M. 2006. Alpha-gliadin genes from the A, B, and D genomes of wheat contain different sets of celiac disease epitopes. BMC Genomics 7:1. 


\section{Electronic Supplementary Material (ESM)}

Electronic Supplementary Material (ESM) associated with this article can be found at the website of CRC at http://www.akademiai.com/content/120427/

Electronic Supplementary Table S1. Parameters and extraction conditions of ELISA assays used in the present study

Electronic Supplementary Table S2. Occurrence of epitope sequences in the storage protein sequences (\%)

Electronic Supplementary Table S3. Number of identified epitope pattern variations (Pattern) and epitope counts (Epitope count) in each sequence in the analyzed prolamin groups of Triticum and Aegilops species. R5 represents hits for the QQPFP peptide, G12 represents hits for the QPQLPY epitope mapping analyses

Electronic Supplementary Figure S1. Normalized R5 and G12 mAb-specific protein content relative to Chinese Spring (\%). Zero point on $\mathrm{x}$ axis represents Chinese Spring, negative values represent lower $\mathrm{mAb}$-specific protein contents, positive values represent higher $\mathrm{mAb}$-specific protein contents 